\title{
Beginning to offer drinking water at birth increases the species richness and the abundance of Faecalibacterium and Bifidobacterium in the gut of preweaned dairy calves
}

\author{
H. K. J. P. Wickramasinghe, ${ }^{1} \oplus$ J. M. Anast, ${ }^{1,2} \odot$ S. Schmitz-Esser, ${ }^{1,2} \odot$ N. V. L. Serão, ${ }^{1}$ \\ and J. A. D. R. N. Appuhamy ${ }^{1 *}$ (1) \\ ${ }^{1}$ Department of Animal Science, lowa State University, Ames 50011 \\ ${ }^{2}$ Interdepartmental Microbiology Graduate Program, lowa State University, Ames 50011
}

\section{ABSTRACT}

We previously demonstrated that dairy calves having access to drinking water since birth (W0) achieved greater body weight, fiber digestibility, and feed efficiency than those that first received drinking water at $17 \mathrm{~d}$ of age (W17). Since gut microbiota composition could be linked to growth and development of animals, the objective of this study was to examine the effect of offering drinking water to newborn calves on composition of bacteria in the gut using a fecal microbiota analysis. Fresh feces were collected directly from the rectum of calves in W0 $(\mathrm{n}=14)$ and W17 $(\mathrm{n}=15)$ at 2,6, and 10 wk of age. All of the calves were fed pasteurized waste milk, weaned at 7 wk of age, and offered tap water according to the treatment. The DNA was sequenced using $16 \mathrm{~S}$ rRNA gene-amplicon sequencing on an Illumina MiSeq system (Illumina Inc., San Diego, CA). The sequences were clustered into operational taxonomic units (OTU) with a $99 \%$ similarity threshold. Treatment effects on $\alpha$-diversity indices and relative abundance of the 10 most abundant genera were analyzed using GLIMMIX procedure of SAS (SAS Institute Inc., Cary, NC). Statistical significance (q-value) of treatment effects on the 50 most abundant OTU was determined with a false discovery rate analysis. At 2 wk of age, W0 had a greater number of observed OTU $(5,908$ vs. 4,698$)$ and species richness (Chao 1 index) than W17. The number of OTU and richness indices increased from wk 2 to 6 , but the increment of W17 was greater than that of W0. The Shannon and inverse-Simpson indices increased linearly with age, but no difference was observed between W0 and W17 at any time point. The Firmicutes to Bacteroidetes ratios were also similar at every time point but decreased markedly when calves were weaned. The relative abun-

Received July 13, 2019.

Accepted January 21, 2020.

*Corresponding author: appuhamy@iastate.edu dance of genera Faecalibacterium and Bacteroides was greater in W0 than W17 at 2 wk of age. The genus Faecalibacterium continued to be more abundant in W0 than W17 at 6 wk of age but had similar abundance 3 wk after weaning (10 wk of age). The abundance of Faecalibacterium at wk 6 was positively correlated with apparent total-tract digestibility of acid detergent fiber at $10 \mathrm{wk}$ of age. Calves receiving water since birth had greater abundance of OTU related to Faecalibacterium prausnitzii, and Bifidobacterium breve at 6 wk of age ( $q$ $<0.085)$. These species are known to improve growth in preweaned calves. The abundance of none of the genera and OTU was different between W0 at W17 at 10 wk of age $(q>0.100)$. Overall, beginning to offer drinking water at birth has a potential to modulate gut microbiota composition and thereby positively affect performance of young dairy heifer calves $(\leq 10 \mathrm{wk}$ of age).

Key words: drinking water, Faecalibacterium, Bifidobacterium

\section{INTRODUCTION}

Even though water is recognized as the most essential nutrient to sustain life and performance of animals, water requirements of livestock species are often overlooked. The situation appears to be even worse for young calves than mature cattle (Beede, 2005; Kertz et al., 2017). The majority of dairy producers tend to refrain from offering drinking water to newborn dairy calves, assuming that water in milk or milk replacers are adequate to fulfil the total water requirement. The USDA's National Animal Health Monitoring System study in 2014 found that dairy producers wait, on average, $17 \mathrm{~d}$ to first offer drinking water to newborn dairy calves (USDA, 2016). In a controlled study, we previously examined the effect of offering drinking water since birth (W0) versus at a later age (17 d of age; W17) on water and feed intake, growth, and nutrient digestibility of young dairy heifer calves (Wick- 
ramasinghe et al., 2019). The W0 group consumed a significant amount of water from buckets $(0.75 \mathrm{~kg} / \mathrm{calf}$ per d) during the first $16 \mathrm{~d}$ of preweaning period. On the other hand, the W17 group consumed 59\% more drinking water than the W0 group once they had access to it during the rest of the preweaning period. Calves offered drinking water from birth achieved greater BW during preweaned period and had improved ADF and NDF digestibility 3 wk after weaning (Wickramasinghe et al., 2019). Growth, nutrient digestibility, and feed efficiency in young calves are significantly related to the development and function of gastrointestinal tract, in which establishment and colonization of microbiota play a key role (Baldwin et al., 2004). Moreover, manipulation of the gut microbiome in the early stage of development is considered a promising way to achieve permanent benefits pertaining to nutrient utilization efficiency in animals (Yáñez-Ruiz et al., 2015).

At birth, the gastrointestinal tract in ruminants is yet to be developed to be fully functional. It is rapidly colonized by diverse communities of microbiota during and immediately after birth (Meale et al., 2016; Yeoman et al., 2018). Several factors including $\mathrm{pH}$, digesta passage rate, and oxygen gradient in the gut greatly affect the number and composition of microbial communities in the digestive tract of calves (Malmuthuge et al., 2015). Water is often offered to young calves in buckets, drinking from which minimally stimulates formation of the esophageal groove. Therefore, unlike milk or milk replacer bypassing the rumen, drinking water receives a chance to be an integral component of the entire gastrointestinal tract and thus affect physiochemical properties of it. Studies using other species suggest that drinking water could affect the composition of gut microbiota by modulating some important physiochemical properties of the gut. For instance, Cremer et al. (2017) reported that drinking water altered digesta passage rate, digesta mixing and absorption of water, and thereby changed the composition of gut microbiota in humans. Sasada et al. (2015) showed that chlorinated water selectively decreased the abundance of some bacterial communities in the human gut. Sofi et al. (2014) suggested that $\mathrm{pH}$ of drinking water affects the composition of gut microflora in mice. Moreover, Faulkner and Weiss (2017) indicated that trace minerals in drinking water could affect the relative abundance of some gut microbial communities.

No study has, however, explored the effect of drinking water on composition of microbiota in the gut of calves or any other group of cattle. Moreover, little is known about potential associations of gut microbiota with growth efficiency in preweaned calves, which has been shown to affect positively future milk produc- tion (Soberon et al., 2012; Van De Stroet et al., 2016). Oikonomou et al. (2013) demonstrated that preweaned calves with increased ADG had a greater bacterial species richness (Chao 1 index) in the gut than those with low ADG as determined with a fecal microbiota analysis. Meale et al. (2016, 2017b) observed significant relationships between $\mathrm{BW}$ and the abundance of several bacterial taxa in fecal microbiota of dairy calves. Moreover, Shanks et al. (2011) and Paz et al. (2018) demonstrated that feed efficiency and nutrient concentrations in feces were different also in mature cattle with different bacterial community compositions in the rumen and feces. We hypothesized that having access to drinking water during the early postnatal period would significantly affect the bacterial community composition in the gut of young dairy calves. The objectives of the present study were (1) to investigate the effect of offering drinking water from birth versus about 2 wk of age on the species richness, abundance, and diversity of bacterial communities in the feces of preweaned calves, and (2) to examine if those effects would be persistent once calves are weaned.

\section{MATERIALS AND METHODS}

\section{Animals, Treatments, and Measurements}

All animal procedures in this study were conducted under approval of the Animal Care and Use Committee at Iowa State University (IACUC 7-17-8570-B). The fecal samples used in the present study were obtained in a calf trial published in Wickramasinghe et al. (2019). More details of the Materials and Methods than what is described below are available in Wickramasinghe et al. (2019). Thirty Holstein heifer calves born in the Iowa State University Dairy from August 22 to October 8 in 2017 were allotted to 2 treatments ( 15 calves per treatment): (1) provision of drinking water at birth (W0), and (2) provision of drinking water $17 \mathrm{~d}$ after birth (W17). However, one calf in W0 became severely ill and had to be euthanized before the end of the study. Therefore, data from 14 calves were used for W0 ( $\mathrm{n}=$ 14). All the calves were housed in individual pens in an indoor calf facility until they were 10 wk old. All calves were bottle-fed with $2.0 \mathrm{~kg}$ of pasteurized waste milk 3 times per day until they were 14 d old. From d 14 to 42 , the milk allowance was increased to $3.0 \mathrm{~kg} / \mathrm{calf}$ per feeding $(9.0 \mathrm{~kg} /$ calf per $\mathrm{d})$. Calves were partially weaned by cutting down daily milk allowance from 9.0 to $3.0 \mathrm{~kg} /$ calf per d on d 42 . Calves were completely weaned on d 49. Once offered, each individual calf had free access to clean drinking water and a grain-based starter ration in separate plastic buckets throughout 
the study (from 0 to $70 \mathrm{~d}$ of age). Approximately 5.0 $\mathrm{g}$ of fresh feces was collected directly from the rectum to sterile bags at 2 and 6 wk of age (preweaning), and at $10 \mathrm{wk}$ of age (postweaning). Fecal samples were flash-frozen in dry ice immediately after collection and then transferred to a freezer at $-80^{\circ} \mathrm{C}$ for storage until further processing.

\section{DNA Extraction}

Fecal samples stored at $-80^{\circ} \mathrm{C}$ were thawed under room temperature while being kept on ice. Approximately $0.25 \mathrm{~g}$ of feces from each replicate was used for DNA extraction using DNeasy PowerLyzer PowerSoil Kit (Qiagen Sciences Inc., Germantown, MD) following the manufacturer's protocol (https://www.qiagen .com/us/resources/resourcedetail?id=e9f9f78a-36ee -4c0f-b91d-ff7aaa6444ef\&lang=en) that included a step involving bead-beating (Fisherbrand Bead Mill 24 Homogenizer, Fisher Scientific, Portsmouth, NH) for mechanical disruption of microbial cells. The DNA was eluted from the column with an elution buffer (included in DNeasy PowerLyzer PowerSoil Kit). Concentrations of DNA were quantified using a NanoDrop 2000 spectrophotometer (Thermo Fisher Scientific, Waltham, MA) and adjusted to 25 to $50 \mathrm{ng}$ of DNA/ $\mu \mathrm{L}$. Samples with adjusted DNA concentrations were loaded to a 96well microtiter plate (Microtiter Microplate, Thermo Fisher Scientific), sealed (Thermo Scientific Nunc Sealing Tapes, Thermo Fisher Scientific), and stored at $-80^{\circ} \mathrm{C}$ until shipping to the DNA sequencing facility (Iowa State University DNA facility, Ames, IA).

\section{Microbiota Sequencing, Sequence Processing, and Analysis}

The microbiota sequencing was conducted using a protocol designed to amplify bacteria and archaea (The Earth Microbiome Project; http://www .earthmicrobiome.org/). Briefly, the genomic DNA from each sample was amplified using Platinum Taq DNA Polymerase (Thermo Fisher Scientific) with one replicate per sample using universal $16 \mathrm{~S}$ rRNA gene bacterial primers [515F (5'-GTGYCAGCMGCCGCGGTAA-3'; Parada et al., 2016) and 806R (5'-GGACTACNVGGGTWTCTAAT-3'; Apprill et al., 2015)] for the variable region V4, as previously described (Kozich et al., 2013). All samples underwent PCR with an initial denaturation step at $94^{\circ} \mathrm{C}$ for $3 \mathrm{~min}$, followed by $45 \mathrm{~s}$ of denaturing at $94^{\circ} \mathrm{C}, 20 \mathrm{~s}$ of annealing at $50^{\circ} \mathrm{C}$, and $90 \mathrm{~s}$ of extension at $72^{\circ} \mathrm{C}$. This was repeated for 35 total PCR cycles and finished with a 10-min extension at $72^{\circ} \mathrm{C}$. All PCR products were then purified with the
QIAquick 96 PCR Purification Kit (Qiagen Sciences Inc., Germantown, MD) according to the manufacturer's recommendations. The PCR bar-coded amplicons were mixed at equal molar ratios and used for Illumina MiSeq paired-end sequencing with 150-bp read length and cluster generation with $10 \%$ PhiX control DNA on an Illumina MiSeq platform (Illumina Inc., San Diego, CA).

Raw sequence data in fastq format were analyzed using mothur v.1.40.4 (Kozich et al., 2013). The number of raw reads per sample varied from 41,076 to 239,819 . Barcode sequences, primer, and low-quality sequences were trimmed using a minimum average quality score of 35 , with a sliding window size of $50 \mathrm{bp}$. Briefly, pairedend reads were combined into contigs using merge.contigs and the sequences were then subsampled randomly to obtain 41,000 sequences per sample and screened for quality with the screen.seqs command excluding sequences shorter than 250 bp and sequences with any ambiguities. The length of allowed homopolymers was set to 8. Chimeras were removed using the chimera. uchime command. The sequences were aligned using the SILVA SSU reference database (version 132, Pruesse et al., 2007). A total of $3,177,001$ sequences $(89.2 \%)$ passed the quality control. These sequences were clustered into operational taxonomic units (OTU) with a $99 \%$ similarity cutoff. The SILVA SSU reference database version 132 was used as taxonomic reference for the OTU. The nonparametric species richness estimates [Chao 1 and abundance-based coverage estimator (ACE)], the diversity indices (Shannon), and the evenness and coverage-based index (Simpson) were calculated using the "summary.single" command. Analysis of $\beta$ diversity based on a Bray-Curtis dissimilarity matrix was achieved by using the analysis of molecular variance and analysis of similarity commands in mothur. Overall variation in bacterial communities was visualized using principal coordinate analysis based on Bray-Curtis dissimilarity. This information was generated with the Phyloseq (v1.28.0, McMurdie and Holmes, 2013) package using the shared and taxonomy file generated in mothur.

Sequencing data are available in the BioProject SRA database under accession number PRJNA526931 (https://www.ncbi.nlm.nih.gov/bioproject/?term= PRJNA526931).

\section{Statistical Analysis}

Treatment effects on $\alpha$ diversity, the abundance of phyla Firmicutes and Bacteroidetes, and the abundance of the 10 most abundant genera were analyzed using GLIMMIX procedure of SAS v. 9.4 (SAS Institute Inc., 
Cary, NC), while accounting for the repeated measures using following model with Poisson distribution for relative abundance data.

$$
\mathrm{Y}_{\mathrm{ijk}}=\mu+\mathrm{T}_{\mathrm{i}}+\mathrm{W}_{\mathrm{j}}+(\mathrm{T} \times \mathrm{W})_{\mathrm{ij}}+\mathrm{C}_{\mathrm{k}}+\mathrm{e}_{\mathrm{ijk}} \text {, }
$$

where $Y_{\mathrm{ijk}}=$ the diversity measure of interest, $\mu=$ overall mean, $\mathrm{T}_{\mathrm{i}}=$ the fixed effect of the ith treatment $(\mathrm{i}=\mathrm{W} 0$ and $\mathrm{W} 17), \mathrm{W}_{\mathrm{j}}=$ the fixed effect of $\mathrm{jth}$ week of age that fecal samples were collected, $(\mathrm{T} \times \mathrm{W})_{\mathrm{ij}}=$ the fixed effect of interaction between the ith treatment and jth week of age, $\mathrm{C}_{\mathrm{k}}=$ the random effect of calf, and $\mathrm{e}_{\mathrm{ijk}}=$ the random error. Relative abundance of OTU was ranked highest to lowest and the 50 most abundant OTU at each time point $(2,6$, and 10 wk of age) were included in the statistical analysis. The treatment effects on each OTU was determined after adjusting the $P$-values into $q$-values using false discovery rate (Benjamini and Hochberg, 1995) correction conducted with MULTTEST procedure in SAS. Statistically significant treatments effects were declared at $q$-value $<0.10$. Additionally, Spearman's rank correlation coefficients were determined for relationships of relative abundance of top 10 genera at wk 6 or at wk 10 with apparent total-tract digestibility of ADF, NDF, starch, and $\mathrm{CP}$ measured at wk 10 using CORR procedure of SAS.

\section{RESULTS}

\section{Alpha Diversity}

Treatment effects on $\alpha$ diversity indices are given in Table 1. At 2 wk of age, W0 had a greater number of observed bacterial species (OTU) as opposed to W17 calves $(P=0.033)$. However, no differences were found at the age of wk 6 or 10 . The number of OTU continued to increase with age in both groups $(P<0.001)$, but the increment from wk 2 to 6 in W17 was more prominent than the increment in W0 (53 vs. 13\%). Consistent with the number of OTU, W0 calves also had greater Chao 1 and ACE indices, indicating improved species richness $(P<0.050)$ relative to $\mathrm{W} 17$ at 2 wk of age. Chao 1 and ACE continued to increase with age $(P<0.001)$ but became similar between $\mathrm{W} 0$ and $\mathrm{W} 17$ at 6 and $10 \mathrm{wk}$ of age. Again, the increment from wk 2 to 6 was more pronounced in W17 (52 and $62 \%$, respectively) than in W0 (8 and $11 \%$, respectively). The Shannon index was similar between W0 and W17 at wk 2 and increased markedly from wk 2 to 6 in both cases $(P<0.001)$. Nonetheless, the W17 calves drinking more water, once offered (Figure 1), had a greater Shannon index than W0 (5.45 vs. $5.09, P=0.029$ ) at wk 6 . Once calves were weaned, Shannon index became similar between W0 and W17. In line with the Shannon's index, the inverse

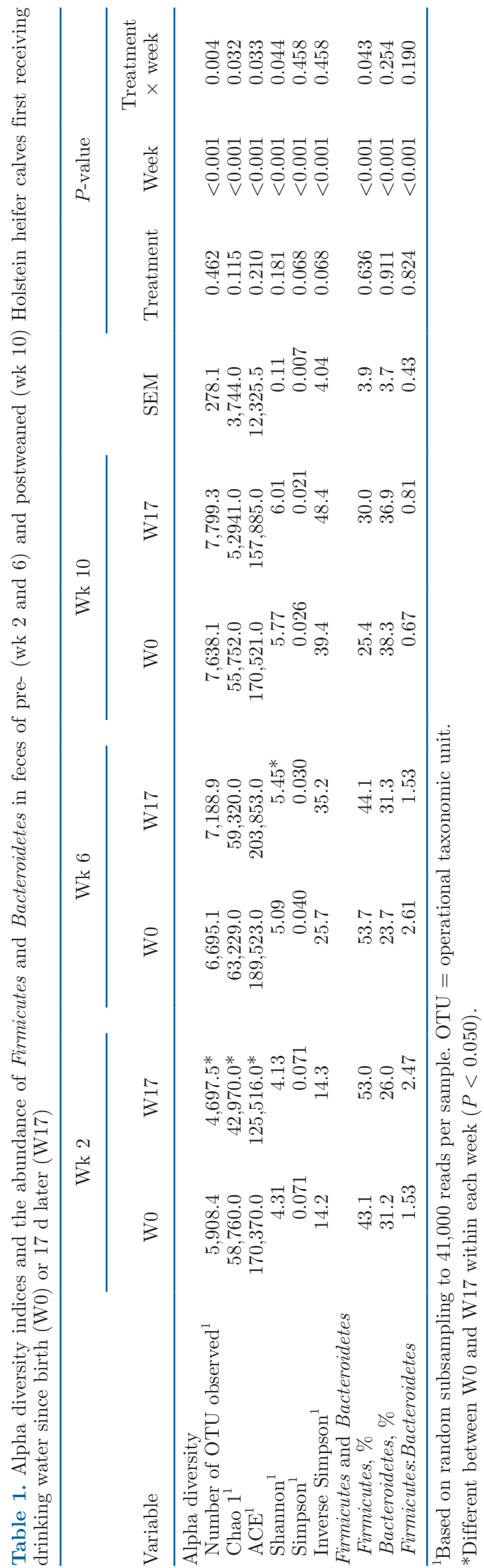




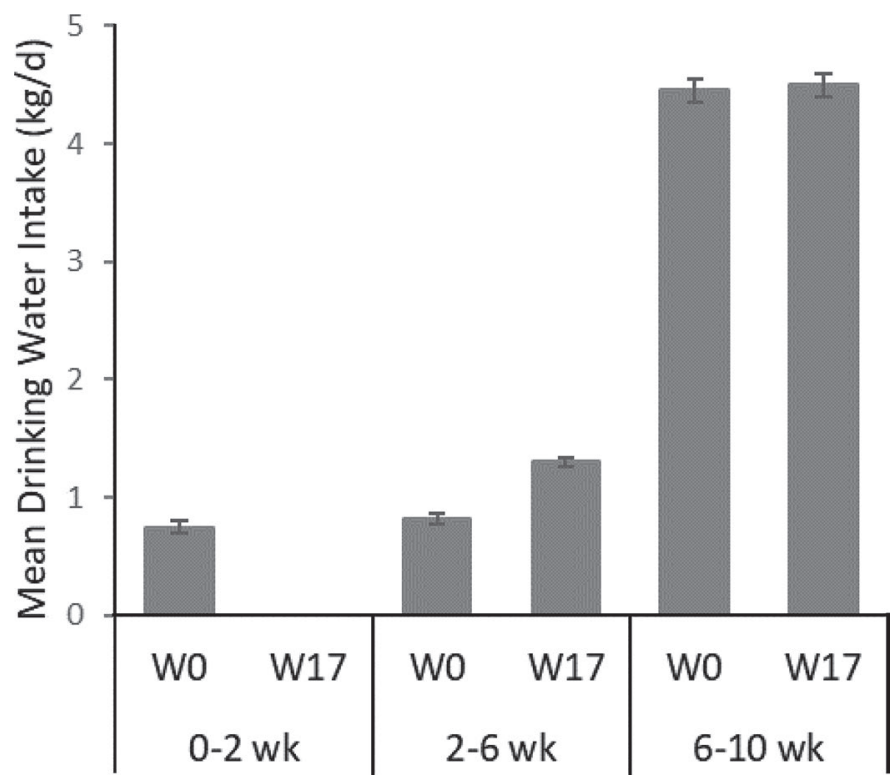

Figure 1. Mean $( \pm$ SEM $)$ drinking water intake of Holstein heifer calves first receiving drinking water at birth (W0) or $17 \mathrm{~d}$ later (W17) during the first $2 \mathrm{wk}, 2$ to $6 \mathrm{wk}$ (preweaning), and 6 to $10 \mathrm{wk}$ (postweaning) of age.

of Simpson index was similar between W0 and W17 at wk $2(P=0.747)$ but tended to be greater in W17 than W0 at wk $6(P=0.055)$ and again became similar between the treatments 3 wk after weaning.

\section{Effects on the Beta Diversity}

When comparing the fecal communities as a whole, no differences were found between W0 and W17 using analysis of molecular variance $(P \geq 0.310$, data not shown) and analysis of similarity $(P \geq 0.251,-0.046$ $<\mathrm{r} \leq 0.026)$. Similarly, principal coordinate analysis based on Bray-Curtis dissimilarities revealed clustering of the samples according to age, but not according to treatment (Figure 2).

\section{Abundance of Firmicutes and Bacteroidetes}

The relative abundance of Firmicutes and Bacteroidetes in $\mathrm{W} 0$ and $\mathrm{W} 17$ at each time point is given in Table 1 and Figure 3. Beginning to offer drinking water at birth versus about 2 wk later did not affect the abundance of Firmicutes or Bacteroidetes and their ratio at any time point $(P>0.150)$. Regardless of the treatments, the abundance of Bacteroidetes and Firmicutes tended to increase and decrease $(P<0.010)$, respectively, as calves were weaned.

\section{Most Abundant Genera and OTU}

Mean $( \pm$ SEM $)$ relative abundance of the dominant genera in W0 and W17 at 2, 6, and 10 wk of age is given in Table 2. Moreover, the mean $( \pm \mathrm{SEM})$ abundance of the top 50 OTU exhibiting acceptable false discovery rate $(q<0.10)$ is given in Table 3 . Regardless of treatments, the abundance of genera Bacteroides, Faecalibacterium, Lachnospiraceae_UC, Lactobacillus, and Tyzzerella_4 decreased $(P<0.001)$, whereas that of Muribaculaceae_GE, Prevotella_1, Ruminococcaceae_UCG-005, and Treponema_2 increased $(P<$ 0.001 ) as calves grew from 2 to 10 wk of age. The most abundant genera and OTU responded to treatments only during the preweaning period ( $<7 \mathrm{wk}$ of age). The abundance of none of the genera or OTU was different

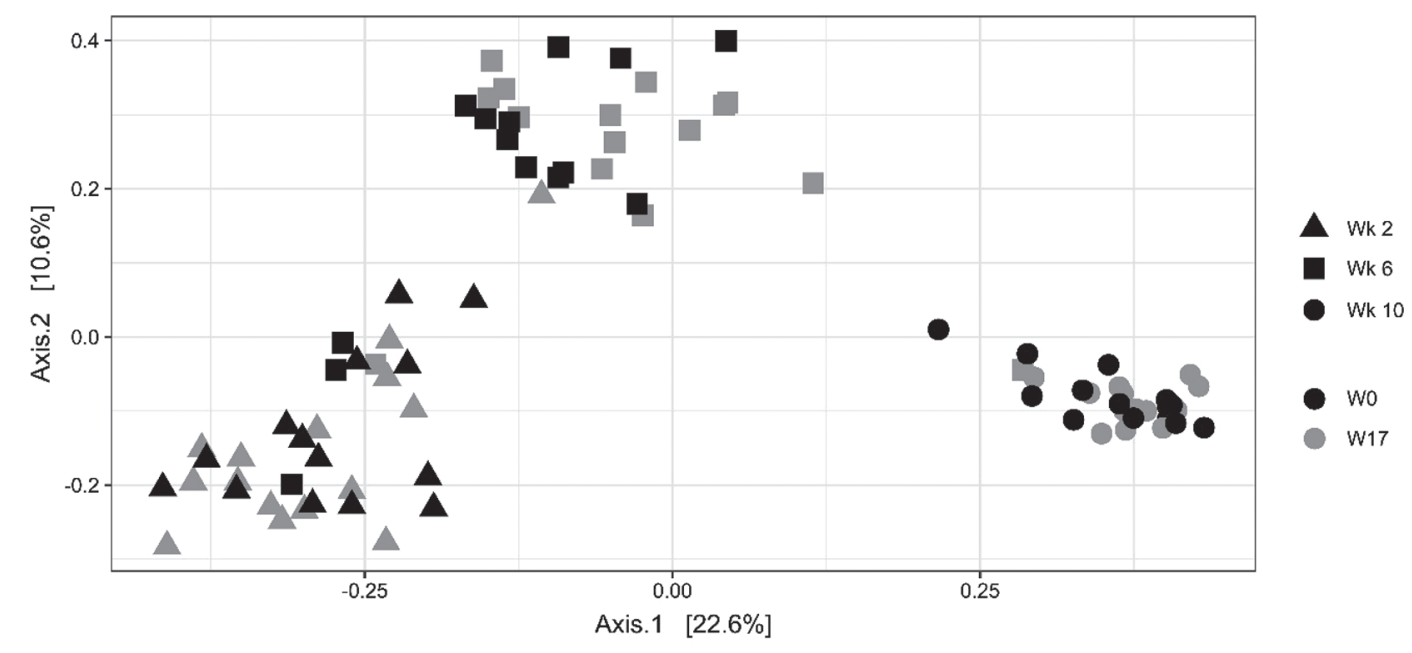

Figure 2. Beta diversity of fecal microbial communities at 3 different time points in response to beginning to offer water to calves from birth (W0) or $17 \mathrm{~d}$ of age (W17). The plot is based on Bray-Curtis differences. 


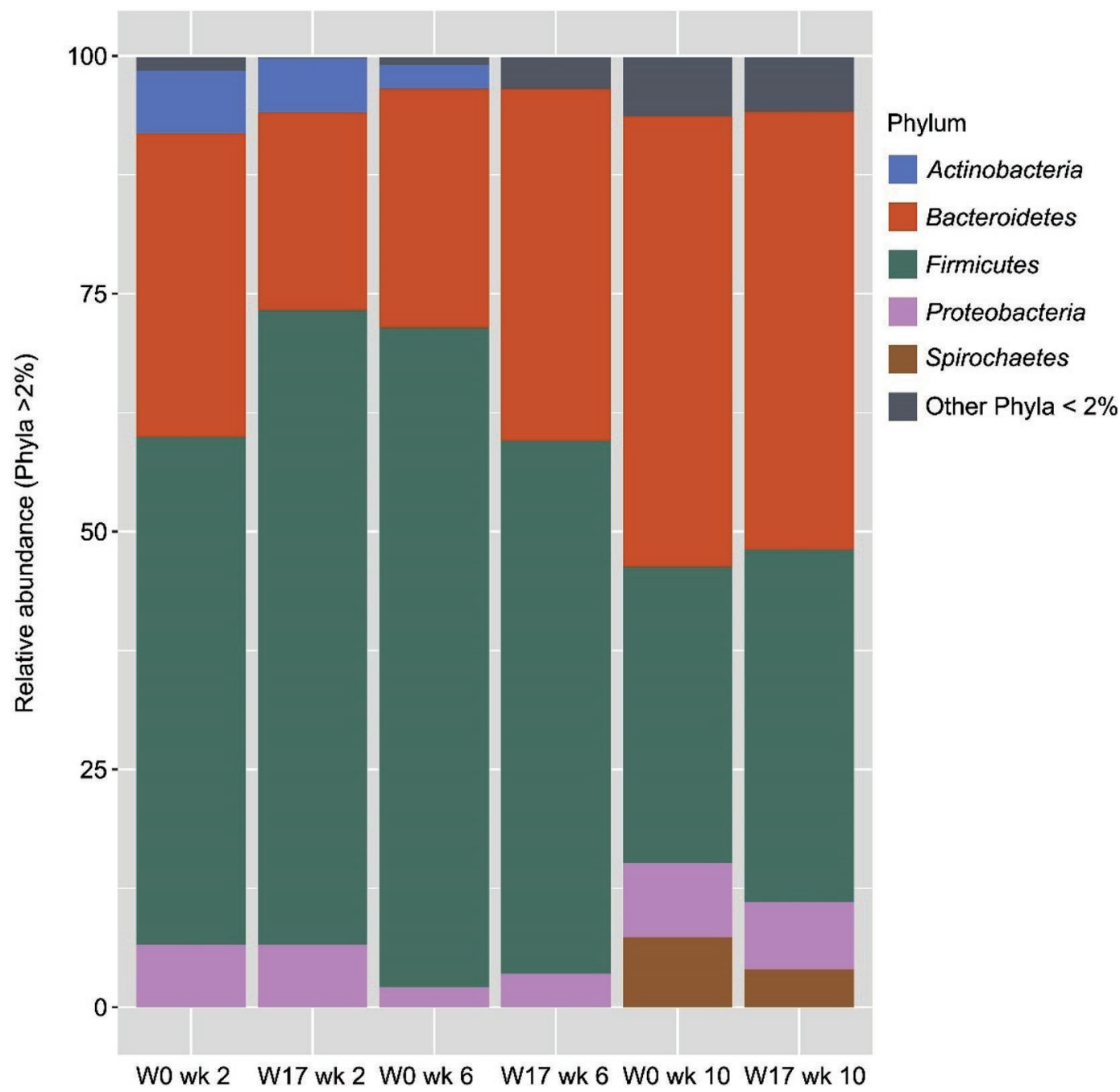

Figure 3. Median relative abundance of bacterial phyla in feces from calves first offered drinking water at birth (W0) or $17 \mathrm{~d}$ later (W17) at 2, 6 (preweaning), and 10 (postweaning) wk of age. Phyla with relative abundance lower than $2 \%$ are shown as "other phyla."

between W0 and W17 few weeks after weaning (10 wk of age).

2 Wk of Age. Genera Bacteroides and Faecalibacterium were more abundant in W0 than $\mathrm{W} 17(P<0.050)$, whereas the abundance of Lachnospiraceae_UC was 2 times greater in W17 than W0 $(P=0.006$, Table 2$)$. Among 50 most abundant OTU, Bacteroides_OTU05 $(q=0.001)$, Bacteroides_OTU37 $(q=0.009)$, and Faecalibacterium_OTU11 $(q=0.056)$ were more abundant in W0 than W17. On the other hand, the abundance of Streptococcus_OTU12 $(q<0.001)$, Lachnospiraceae_OTU09 $(q=0.001)$, Lachnospiraceae_OTU28 ( $q$ $=0.066)$, and Butyricicoccus_OTU15 $(q=0.056)$ was greater in W17 calves than W0 calves (Table 3).

6 Wk of Age. Genus Faecalibacterium continued to be more abundant in W0 than W17 $(P=0.025)$, whereas the abundance of genus Muribaculaceae was greater in $\mathrm{W} 17$ than $\mathrm{W} 0(P=0.041$, Table 2$)$. Among the 50 most abundant OTU, the abundance of Lactobacillus_OTU01 $(q=0.049)$, Tyzzerella_OTU04 $(q=$ 0.049), Streptococcus_OTU12 $(q=0.046)$, Faecalibacterium_OTU08 $(q=0.019)$, Bifidobacterium_OTU14 $(q=0.082)$, and Lachnospiraceae_OTU28 $(q=0.060)$ were different between W0 and W17. All those 6 OTU were more abundant in W0 than W17 (Table 3).

\section{Relationships Between Gut Microbiota Composition and Nutrient Digestibility}

Correlations ( $\mathrm{r} ; \mathrm{r}=$ Spearman's rank correlation coefficients) of the abundance of dominant genera at wk 6 or 10 with apparent total-tract digestibility of $\mathrm{ADF}, \mathrm{NDF}$, starch, and $\mathrm{CP}$ at wk 10 are given in Table 4. The abundance of Faecalibacterium preweaning was 


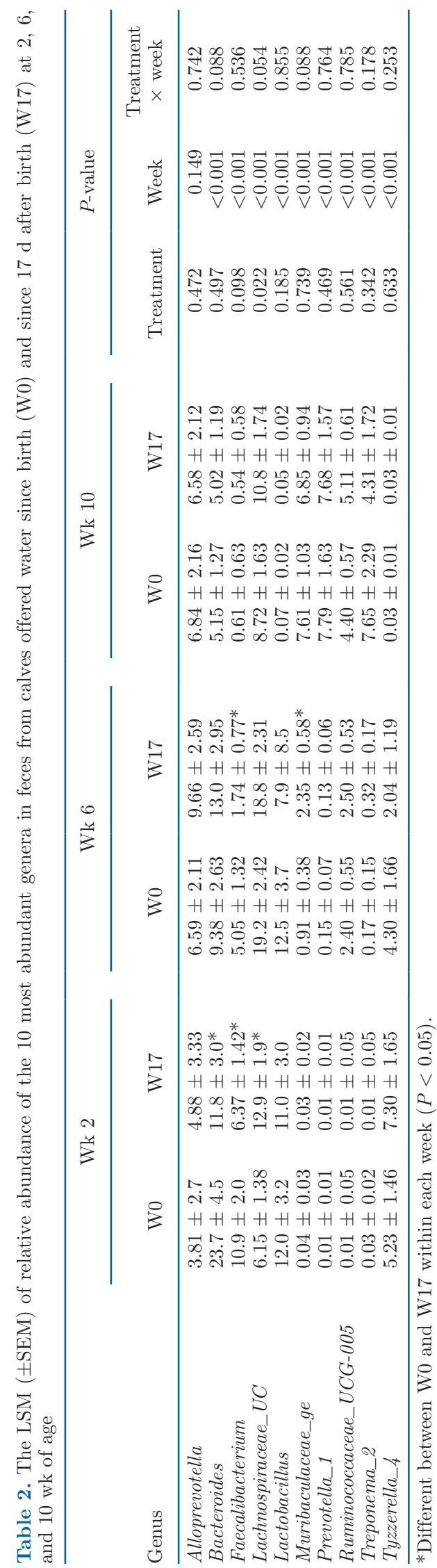

positively related to the ADF digestibility postweaning $(\mathrm{R}=0.45, P=0.017)$, whereas the abundance of Faecalibacterium postweaning was negatively related to the digestibility of starch postweaning $(\mathrm{R}=-0.40, P$ $=0.037$ ). Weaned calves having greater abundance of Lactobacillus were related to lower digestibility of ADF $(\mathrm{R}=-0.45, P=0.016)$. Treponema $\_2$ had a negative relationships with the ADF digestibility $(\mathrm{R}=-0.44, P$ $=0.018)$, whereas Prevotella $\_1$ had a positively relationship with NDF digestibility $(\mathrm{R}=0.41, P=0.029)$ 3 wk postweaning.

\section{DISCUSSION}

Pertaining to the fact that dairy producers wait, on average, $17 \mathrm{~d}$ to first offer drinking water to newborn dairy calves in the United States (USDA, 2016), we studied the effect of beginning to offer drinking water at birth (W0) versus at $17 \mathrm{~d}$ of age (W17) on growth, health, and nutrient digestibility, and fecal microbiota community composition. The effects on all those variables except the fecal microbiota composition were published in Wickramasinghe et al. (2019). The present paper includes the effects on fecal microbiota as measured at 2, 6 (preweaning), and $10 \mathrm{wk}$ (early postweaning) of age. In line with several previous investigations (Callaway et al., 2010; McGarvey et al., 2010; Mao et al., 2012; Oikonomou et al., 2013; Kim et al., 2014; Klein-Jöbstl et al., 2014), we chose to study fecal microbiota as a proxy for gut microbiota as fecal samples make periodic measurements with adequate sample size possible. One major limitation of using fecal samples is the fact that fecal microbiota primarily reflects digesta-associated microbiota, which can be markedly different from microbiota found in the mucosa (Malmuthuge et al., 2012, 2014). Moreover, fecal samples are highly representative of the digesta in the lower gut and would not adequately represent important hostmicrobial interactions specific to different regions of the gut (Malmuthuge et al., 2014). Even though fecal microbiota analyses may not adequately describe interactions between gut microbiota and host physiology, they can still be able to capture changes in gut microbiota composition (Pereira et al., 2016). In this study, we describe the gut microbiota composition primarily using the relative abundance data, which would limit drawing robust conclusions on absolute prevalence of bacterial taxa. Therefore, the present fecal microbiota analysis was focused on examining relative differences in microbiota composition in calves receiving drinking water from birth versus $17 \mathrm{~d}$ later. Furthermore, correlations between nutrient digestibility (postweaning) and gut microbiota composition (pre- or postweaning) were examined with an aim of explaining any lasting 
Table 3. The LSM $( \pm \mathrm{SEM})$ of relative abundance of only operational taxonomic units (OTU) showing treatment effects $(q<0.10)$ according to a false discovery rate analysis ${ }^{1}$

\begin{tabular}{lccc}
\hline OTU & W0 & W17 & $q$-Value \\
\hline Wk 2 & & & \\
Bacteroides_OTU05 & $8.80 \pm 0.80$ & $3.21 \pm 0.47$ & 0.001 \\
Lachnospiraceae_OTU09 & $2.33 \pm 0.41$ & $6.03 \pm 0.64$ & 0.001 \\
Faecalibacterium_OTU11 & $4.03 \pm 0.54$ & $2.20 \pm 0.39$ & 0.056 \\
Butyricicoccus_OTU15 & $1.96 \pm 0.15$ & $3.70 \pm 0.25$ & 0.056 \\
Streptococcus_OTU12 & $0.37 \pm 0.15$ & $3.68 \pm 0.54$ & $<0.001$ \\
Bacteroides_OTU37 & $2.35 \pm 0.42$ & $0.56 \pm 0.20$ & 0.009 \\
Lachnospiraceae_OTU28 & $0.75 \pm 0.24$ & $1.89 \pm 0.36$ & 0.066 \\
Wk 6 & & & 0.049 \\
Lactobacillus_OTU01 & $6.45 \pm 0.47$ & $3.78 \pm 0.26$ & 0.049 \\
Tyzzerella_OTU04 & $2.68 \pm 0.44$ & $1.02 \pm 0.27$ & 0.046 \\
Streptococcus_OTU12 & $2.35 \pm 0.78$ & $0.74 \pm 0.42$ & 0.019 \\
Faecalibacterium_OTU08 & $2.05 \pm 0.41$ & $0.20 \pm 0.12$ & 0.082 \\
Bifidobacterium_OTU14 & $1.21 \pm 0.31$ & $0.24 \pm 0.13$ & 0.06 \\
Lachnospiraceae_OTU28 & $0.62 \pm 0.28$ & $0.10 \pm 0.09$ & \\
\hline
\end{tabular}

${ }^{1} \mathrm{~W} 0=$ calves receiving drinking water from birth; $\mathrm{W} 17=$ calves first receiving drinking water at $17 \mathrm{~d}$ of age.

effects of offering drinking water to newborn calves on the gut microbiota composition and nutrient utilization efficiencies.

A greater number of OTU was observed in W0 than W17 at 2 wk of age, indicating a positive effect of beginning to offer drinking water at birth on establishment and early colonization of bacteria in the gut of neonatal calves. In addition, the Shannon index was greater in W17 drinking $>50 \%$ more water $(\mathrm{L} / \mathrm{d})$ than $\mathrm{W} 0$ at 6 wk of age, suggesting a positive effect of drinking water volume on gut microbiota diversity. Malmuthuge et al. (2015) describe that early colonization of microbiota in the gut of dairy calves is influenced by factors related to host (e.g., luminal $\mathrm{pH}$ and digesta passage rates), the environment (e.g., diet and antimicrobial treatments), and microbiota (e.g., mechanisms to survive under oxygen gradient and to obtain nutrients from the host). In a pursuit of explaining the differences in species richness between W0 and W17, one could speculate that bacteria present in drinking water itself might have contributed to gut microbiota communities to a certain extent. However, Ling et al. (2018) reported that aerobic bacteria (e.g., Proteobacteria) were the most abundant $(>50 \%)$, whereas anaerobic bacteria (e.g., Bacteroidetes and Firmicutes) had negligible contribution $(<3 \%)$ to the bacterial community composition of tap water. Given the anaerobic environment of the gastrointestinal tract, even if gut microbiota could be inoculated with the microbiota in drinking water, resulting compositional changes would be rather transient. On the other hand, those short-lived aerobic bacteria deriving from drinking water could still have an effect on gut microbiota possibly by promoting colonization of anaerobic bacteria, as the aerobic bacteria would scavenge on oxygen in the gut, thereby making it adequately anaerobic (Friedman et al., 2018). Among other factors, the host diet and nutrients available for gut microbiota are a major consideration (Dill-McFarland et al., 2019). Milk ( $\sim 800 \mathrm{~g}$ of milk solid/d) was the primary contributor to nutrients available in the gut as

Table 4. Spearman's rank correlation coefficients for the relationships of the abundance of the top 10 genera at 6 wk of age (preweaning) and 10 wk of age (3 wk postweaning) with apparent total-tract digestibility of ADF, NDF, starch, and CP measured at 10 wk of age (3 wk postweaning)

\begin{tabular}{|c|c|c|c|c|c|c|c|c|}
\hline \multirow[b]{2}{*}{ Genus } & \multicolumn{4}{|c|}{ Abundance at wk 6 (preweaning) } & \multicolumn{4}{|c|}{ Abundance at wk 10 (postweaning) } \\
\hline & $\mathrm{ADF}$ & $\mathrm{NDF}$ & Starch & $\mathrm{CP}$ & $\mathrm{ADF}$ & NDF & Starch & $\mathrm{CP}$ \\
\hline Bacteroides & 0.10 & 0.05 & 0.06 & -0.31 & -0.25 & -0.24 & 0.3 & 0.28 \\
\hline Faecalibacterium & $0.45^{*}$ & -0.01 & -0.21 & 0.19 & 0.08 & -0.15 & $-0.40^{*}$ & -0.33 \\
\hline Lachnospiraceae_UC & -0.12 & 0.16 & 0.13 & 0.33 & 0.05 & 0.05 & -0.22 & -0.07 \\
\hline Lactobacillus & -0.31 & -0.35 & 0.14 & -0.10 & $-0.45^{*}$ & -0.34 & -0.16 & -0.12 \\
\hline Ruminococcaceae_UCG-005 & -0.03 & -0.10 & 0.05 & -0.07 & 0.07 & 0.08 & -0.11 & -0.27 \\
\hline Treponema_2 & -0.03 & -0.01 & 0.06 & -0.24 & $-0.44^{*}$ & -0.34 & 0.09 & 0.24 \\
\hline Tyzzerella_4 & 0.01 & 0.32 & 0.17 & 0.38 & -0.02 & 0.33 & -0.04 & -0.09 \\
\hline
\end{tabular}

*Statistically significant $(P<0.05)$. 
solid feed intake was negligible at $20 \mathrm{~g} / \mathrm{d}$ in both W0 and W17 during the first 2 wk (Wickramasinghe et al., 2019). Calves having access to drinking water, however, consumed 6\% (Wickramasinghe et al., 2019) more milk than W17 during this period. Given the fact that milk bypasses the rumen and is shunted to the abomasum, it can be hypothesized that the increment in milk intake and thus nutrient supply to the intestines could be associated with a significant enhancement of colonization bacteria and thus the species richness in the lower gut. The average milk intake $(\mathrm{kg} / \mathrm{d})$ during the first $2 \mathrm{wk}$ was, however, inversely related to the Shannon index, an indicator of the evenness of microbiota composition at 2 wk of age $(\mathrm{R}=-0.55, P=0.002$, data not shown), suggesting that the increment of milk intake likely supported colonization of only certain bacterial taxa. On the other hand, even though nutrient intake is constant, changes in digestion kinetics would diversify amounts and the forms of nutrients available in whole or a particular segment of the digestive tract. Passage rate is a key factor that would satisfactorily explain variability in digestibility of nutrients. High passage rates interfere with digestibility and result in elevated outflow of nutrients in varying forms (e.g., undigested nutrients and intermediary products of digestion), promoting growth of a variety of bacteria with diversified substrate preferences. We collected total feces output, analyzed nutrient concentrations in feces, and determined apparent total-tract digestibility of nutrients only of weaned calves (10 wk of age, Wickramasinghe et al., 2019). Such information of younger calves could have been useful in explaining the observed differences in the diversity of gut microbiota between W0 and W17 at wk 2 and 6. Nonetheless, Fraley et al. (2015) demonstrated that dairy cows consuming high drinking water volume $(\mathrm{L} / \mathrm{d})$ were related to high liquid passage rates from the rumen and low concentrations of VFA and ammonia in the rumen. Therefore, it can be hypothesized that water entering the rumen would elevate intraluminal pressure in the rumen, which in turn stimulates the pressure receptors in rumen wall and thus rumen contractions (Forbes and Barrio, 1992). Little is known about the role of intraluminal pressure in preruminants, but it is known to stimulate gastric emptying in monogastric animals (Strunz and Grossman, 1978). Therefore, perhaps, calves drinking more water were associated with greater digesta passage rates and thus increased nutrient flows to the lower gut, promoting colonization of bacteria in it.

It can also be speculated that drinking water might modulate physiochemical properties of the gut, for instance $\mathrm{pH}$ and redox potential, and thereby influence the composition of gut microbiota in neonatal calves. Even though it is hard to find any scientific evidence supporting such a notion for calves, Fraley et al. (2015) observed a positive correlation between drinking water intake and fecal $\mathrm{pH}$ in dairy cows. Reese and Dunn (2018) reported a positive correlation between gut $\mathrm{pH}$ and the Shannon index of gut microbiota across several species. Moreover, drinking water consumed by dairy cattle is not completely nutrient free where mineral concentrations are concerned (Castillo et al., 2013). Mineral supplements have been reported to shape the diversity of intestinal microbiota in young monogastric animals (Shannon and Hill, 2019). Faulkner and Weiss (2017) showed that trace minerals such as copper, zinc, and manganese significantly alter the relative abundance of microbial communities in the colon of lactating dairy cows. It is also noteworthy that calves in the present study were offered chlorinated tap water and fed pasteurized waste milk, often containing residues of various antimicrobial substances. (Pereira et al., 2014; Tempini et al., 2018). Xi et al. (2009) reported that chlorine in drinking water increased expression of multidrug efflux pumps in bacteria, making them resistant to antibiotics. Perhaps, this possible interaction between drinking water and waste milk had a more favorable effect on richness and diversity of gut microbiota in W0 than W17.

At the phylum level, offering drinking water from birth or about 2 wk later did not change relative abundance of Firmicutes and Bacteroidetes, or their ratio in young calves ( $\leq 10 \mathrm{wk}$ of age). However, the abundance significantly changed with age, particularly when calves were weaned, in agreement with the previously reported relationship with the development of gastrointestinal tract and weaning of young calves (Meale et al., 2017a,b). At the genus level, Alloprevotella, Bacteroides, Faecalibacterium, Lachnospiraceae, Lactobacillus, Muribaculaceae, Prevotella_1, Ruminococcaceae_UCG-005, Treponema_2, and Tyzzerella_4 were the most abundant during the first $10 \mathrm{wk}$. This list is not perfectly in line with the lists published previously for calves (e.g., Oikonomou et al., 2013 and Meale et al., 2017b) due to several factors including age, diet, weaning strategies, and geographical location. In addition, as mentioned above, calves in the present study were fed waste milk potentially containing antimicrobial residues, which are able to modulate the composition of gut microbiota differently. Nonetheless, relative abundance of the majority of genera changed significantly as the calves got older and were weaned. Furthermore, the abundances of every genera were highly variable across individual animals at every time point $(\mathrm{CV}>45 \%$, data not shown). Overall, as also observed in Figure 2, the individual animal variability was greater preweaning than postweaning (average CV $=163$ vs. $109 \%$, data not shown). With that much of a variability, we were still 
be able to capture treatment differences $(P<0.050)$ of some genera, indicating the sample size of this study $(\mathrm{n}=14$ to 15$)$ was adequate to capture true effects of drinking water on some important attributes of gut microbiota composition. Provision of drinking water from birth had a positive effect on the abundance of Bacteroides and Faecalibacterium, whereas it negatively affected the abundance of Lachnospiraceae_UC at $2 \mathrm{wk}$ of age. Considering their preference for sugars and oligosaccharides present in milk (Marcobal et al., 2011; Azcarate-Peril et al., 2017), the increased abundance of Bacteroides and Faecalibacterium in W0 could be related to the increased volume of milk they consumed and the capacity of drinking water to enhance liquid passage rates and thus increase nutrients flows to the lower gut.

Among the most abundant OTU, Streptococcus_ OTU12, which was related ( $100 \%$ similarity) to Streptococcus gallolyticus ATCC BAA249 (formerly known as Streptococcus bovis), was less abundant in W0 than W17 at 2 wk of age. Having a low abundance of $S$. gallolyticus in the gut at 2 wk of age could be an advantage as Aydin et al. (2019) found recently a positive association between the $S$. gallolyticus and meningitis in neonatal calves. The inverse association between $S$. gallolyticus and drinking water appeared to be more an effect of drinking water volume $(\mathrm{L} / \mathrm{d})$ than the age calves were first offered drinking water as W17 drinking more water had a lower abundance of $S$. gallolyticus than W0 at 6 wk of age. Meale et al. (2017b) also reported a negative correlation between drinking water volume (L/d) and the abundance of genus Streptococcus in feces of dairy calves 5 to 9 wk of age. On the other hand, the abundance of genus Faecalibacterium continued to be more dominant in W0 than W17 even at wk 6, exemplifying a lasting effect of receiving drinking water during first $2 \mathrm{wk}$ of life on gut microbiota composition in calves. In particular, the abundance of Faecalibacterium_OTU08 related (98.8\% similarity) to Faecalibacterium prausnitzii ATCC 27768 was 10 times greater in W0 than W17 at 6 wk of age. Faecalibacterium prausnitzii is an obligate anaerobic, gram-positive, and butyrate-producing bacterium shown to improve preweaned calf performance (Foditsch et al., 2015). For instance, Oikonomou et al. (2013) reported that $F$. prausnitzii in feces was positively related to ADG in preweaned calves. Moreover, Foditsch et al. (2015) showed that feeding $F$. prausnitzii as a probiotic improved gastrointestinal health and growth of preweaned calves. Another OTU that was more prominent in W0 than W17 at 6 wk of age was Bifidobacterium_OTU14 related (100\% similarity) to Bifidobacterium breve ATCC 15700. Bifidobacterium species in the gut pro- vide several benefits to animals, including antagonistic effects against pathogenic bacteria and improvements in ADG (Abe et al., 1995; Simmering and Blaut, 2001; Vaughan et al., 2002; Chierici et al., 2003; Paggi and Fay 2004; Vlková et al., 2004). Meale et al. (2017b) reported a negative relationship between drinking water intake and the abundance of the genus Bifidobacterium in feces of 5- to 9-wk-old dairy calves. Moreover, Owusu-Asiedu et al. (2006) observed that gut Bifidobacteria counts were negatively related to digesta passage rates in growing pigs. Therefore, the differences in the abundance of Bifidobacterium between W0 and W17 appear to be related to the differences in drinking water volumes and subsequent digesta passage rates between those 2 groups at wk 6 .

None of the $\alpha$ diversity indices or the abundance of any of the genera or the OTU responding to drinking water during preweaning period was significantly different between W0 and W17 3 wk postweaning (10 wk of age), suggesting that the drinking-water-induced changes in gut microbiota composition were rather short lived and limited primarily to suckling calves. This also points out the robustness of solid feed in shaping gut microbiota composition compared with other factors. Nonetheless, the abundance of the genus Faecalibacterium that was more prominent in W0 than W17 preweaning (at wk 6) was positively correlated with ADF digestibility 3 wk postweaning (10 wk of age), supporting an idea that early-life changes in gut microbiota composition could be linked to future feed conversion efficiency of calves (Wickramasinghe et al., 2019). The observed relationships between gut microbiota and nutrient digestibility further indicated that weaned calves still having significant abundance of milk sugar-fermenting bacteria likely indicate a lack of adaptation to use a solid diet as evident by a negative correlation between Lactobacillus and ADF digestibility 3 wk postweaning. Moreover, Treponema_2, which is usually dominant in weaned calves (Meale et al., 2016), was also negatively related to ADF and NDF digestibility 3 wk postweaning. Cunha et al. (2017) also reported that Treponema_2 in the rumen were negatively correlated with enteric methane production, which positively responds to NDF digestibility in mature cattle (Appuhamy et al., 2016).

\section{CONCLUSIONS}

Even though the exact mechanism is not clear, offering drinking water from birth likely enhanced establishment and early colonization of bacteria in the gut of newborn calves as indicated by improved microbiota richness indices of W0 at 2 wk of age. Independent of 
the timing that drinking water was first offered, drinking water intake $(\mathrm{L} / \mathrm{d})$ also appeared to have a positive association with diversity indices. Those improvements were, however, limited to preweaned calves. Regardless of the changes in drinking water intake between W0 and W17, W0 had increased abundance of genus Faecalibacterium throughout the preweaning period $(<7 \mathrm{wk}$ of age). Moreover, the abundance of Faecalibacterium was positively related to digestibility of ADF $3 \mathrm{wk}$ after weaning, suggesting a positive link between offering drinking water from birth and ability to use a solid diet efficiently once calves are weaned. The improved growth performance of W0 in our previously published work appeared to be partly related to changes in gut microbiota composition as W0 had increased abundance of Faecalibacterium prausnitzii, and Bifidobacterium breve known to improve growth of preweaned calves. Overall, beginning to offer drinking water to newborn calves at birth could potentially affect gut microbiota composition and thereby have a favorable effect on growth and feed conversion efficiency during early stage of development ( $\leq 10 \mathrm{wk}$ of age). It is, however, noteworthy that we evaluated the associations between drinking water and bacterial taxa using semiquantitative relative abundance data that would possibly limit robustness of some of our conclusions as compared with those based on data from quantitative analysis such as quantitative PCR. Furthermore, since our calves were fed waste milk possibly containing residues of various antimicrobial substances that can affect gut microbiota by themselves or through an interaction with drinking water, the present study results have to be cautiously extrapolated to other calf populations.

\section{ACKNOWLEDGMENTS}

The assistance received from staff at the Calf Unit of the Dairy Research and Teaching Farm at Iowa State University is gratefully acknowledged. The authors have not stated any conflicts of interest.

\section{REFERENCES}

Abe, F., N. Ishibashi, and S. Shimamura. 1995. Effect of administration of Bifidobacteria and lactic acid bacteria to newborn calves and piglets. J. Dairy Sci. 78:2838-2846. https://doi.org/10.3168/ jds.S0022-0302(95)76914-4.

Apprill, A., S. McNally, R. Parsons, and L. Weber. 2015. Minor revision to V4 region SSU rRNA 806R gene primer greatly increases detection of SAR11 bacterioplankton. Aquat. Microb. Ecol. 75:129-137. https://doi.org/10.3354/ame01753.

Appuhamy, J. A. D. R. N., J. France, and E. Kebreab. 2016. Models for predicting enteric methane emissions from dairy cows in North America, Europe, and Australia and New Zealand. Glob. Change Biol. 22:3039-3056. https://doi.org/10.1111/gcb.13339.

Aydin, F., V. Güneş, L. C. Bayram, S. Abay, E. Karakaya, K. Varol, G. Ekinci, K. S. Gümüşsoy, H. K. Müştak, and K. S. Diker. 2019.
Neonatal calf meningitis associated with Streptococcus gallolyticus ssp. gallolyticus. Folia Microbiol. (Praha) 64:223-229. https://doi .org/10.1007/s12223-018-0649-5.

Azcarate-Peril, M. A., A. J. Ritter, D. Savaiano, A. MonteagudoMera, C. Anderson, S. T. Magness, and T. R. Klaenhammer. 2017. Impact of short-chain galactooligosaccharides on the gut microbiome of lactose-intolerant individuals. Proc. Natl. Acad. Sci. USA 114:E367-E375. https://doi.org/10.1073/pnas.1606722113.

Baldwin, R. L., VI, K. R. McLeod, J. L. Klotz, and R. N. Heitmann. 2004. Rumen development, intestinal growth and hepatic metabolism in the pre- and post-weaning ruminant. J. Dairy Sci. 87(E Suppl.):E55-E65.

Beede, D. K. 2005. The most essential nutrient: Water. Pages 13-31 in Proc. 7th Western Dairy Management Conf., Reno, NV. Western Dairy Management Conference, Manhattan, KS.

Benjamini, Y., and Y. Hochberg. 1995. Controlling the false discovery rate: A practical and powerful approach to multiple testing. J. R. Stat. Soc. B 57:289-300. https://doi.org/10.1111/j.2517-6161.1995 .tb02031.x.

Callaway, T. R., S. E. Dowd, T. S. Edrington, R. C. Anderson, N. Krueger, N. Bauer, P. J. Kononoff, and D. J. Nisbet. 2010. Evaluation of bacterial diversity in the rumen and feces of cattle fed different levels of dried distillers grains plus solubles using bacterial tag-encoded FLX amplicon pyrosequencing. J. Anim. Sci. 88:3977-3983. https://doi.org/10.2527/jas.2010-2900.

Castillo, A. R., N. R. St-Pierre, N. Silva del Rio, and W. P. Weiss. 2013. Mineral concentrations in diets, water, and milk and their value in estimating on-farm excretion of manure minerals in lactating dairy cows. J. Dairy Sci. 96:3388-3398. https://doi.org/10 $.3168 /$ jds.2012-6121.

Chierici, R., S. Fanaro, D. Saccomandi, and V. Vigi. 2003. Advances in the modulation of the microbial ecology of the gut in early infancy. Acta Paediatr. Suppl. 91:56-63. https://doi.org/10.1111/j .1651-2227.2003.tb00647.x.

Cremer, J., M. Arnoldini, and T. Hwa. 2017. Effect of water flow and chemical environment on microbiota growth and composition in the human colon. Proc. Natl. Acad. Sci. USA 114:6438-6443. https://doi.org/10.1073/pnas.1619598114.

Cunha, C. S., C. M. Veloso, M. I. Marcondes, H. C. Mantovani, T. R. Tomich, L. G. R. Pereira, M. F. L. Ferreira, K. A. Dill-McFarland, and G. Suen. 2017. Assessing the impact of rumen microbial communities on methane emissions and production traits in Holstein cows in a tropical climate. Syst. Appl. Microbiol. 40:492-499. https://doi.org/10.1016/j.syapm.2017.07.008.

Dill-McFarland, K. A., P. J. Weimer, J. D. Breaker, and G. Suen. 2019. Diet influences early microbiota development in dairy calves without long-term impacts on milk production. Appl. Environ. Microbiol. 85:e02141-18.

Faulkner, M. J., and W. P. Weiss. 2017. Effect of source of trace minerals in either forage- or by-product-based diets fed to dairy cows: 1. Production and macronutrient digestibility. J. Dairy Sci. 100:5358-5367. https://doi.org/10.3168/jds.2016-12095.

Foditsch, C., R. V. V. Pereira, E. K. Ganda, M. S. Gomez, E. C. Marques, T. Santin, and R. C. Bicalho. 2015. Oral administration of Faecalibacterium prausnitzii decreased the incidence of severe diarrhea and related mortality rate and increased weight gain in preweaned dairy heifers. PLoS One 10:e0145485. https://doi.org/ 10.1371/journal.pone.0145485.

Forbes, J. M., and J. P. Barrio. 1992. Abdominal chemo- and mechanosensitivity in ruminants and its role in the control of food intake. Exp. Physiol. 77:27-50. https://doi.org/10.1113/expphysiol .1992.sp003581.

Fraley, S. E., M. B. Hall, and T. D. Nennich. 2015. Effect of variable water intake as ediated by dietary potassium carbonate supplementation on rumen dynamics in lactating dairy cows. J. Dairy Sci. 98:3247-3256. https://doi.org/10.3168/jds.2014-8557.

Friedman, E. S., K. Bittinger, T. V. Esipova, L. Hou, L. Chau, J. Jiang, C. Mesaros, P. J. Lund, X. Liang, G. A. FitzGerald, M. Goulian, D. Lee, B. A. Garcia, I. A. Blair, S. A. Vinogradov, and G. D. Wu. 2018. Microbes versus chemistry in the origin of the 
anaerobic gut lumen. Proc. Natl. Acad. Sci. USA 115:4170-4175. https://doi.org/10.1073/pnas.1718635115.

Kertz, A. F., T. M. Hill, J. D. Quigley III, A. J. Heinrichs, J. G. Linn, and J. K. Drackley. 2017. 100-year review: Calf nutrition and management. J. Dairy Sci. 100:10151-10172. https://doi.org/10.3168/ jds.2017-13062.

Kim, M., J. Kim, L. A. Kuehn, J. L. Bono, E. D. Berry, N. Kalchayanand, H. C. Freetly, A. K. Benson, and J. E. Wells. 2014. Investigation of bacterial diversity in the feces of cattle fed different diets. J. Anim. Sci. 92:683-694. https://doi.org/10.2527/jas.2013-6841.

Klein-Jöbstl, D., E. Schornsteiner, E. Mann, M. Wagner, M. Drillich, and S. Schmitz-Esser. 2014. Pyrosequencing reveals diverse fecal microbiota in Simmental calves during early development. Front. Microbiol. 5:622. https://doi.org/10.3389/fmicb.2014.00622.

Kozich, J. J., S. L. Westcott, N. T. Baxter, S. K. Highlander, and P. D. Schloss. 2013. Development of a dual-index sequencing strategy and curation pipeline for analyzing amplicon sequence data on the MiSeq Illumina sequencing platform. Appl. Environ. Microbiol. 79:5112-5120. https://doi.org/10.1128/AEM.01043-13.

Ling, F., R. Whitaker, M. W. LeChevallier, and W.-T. Liu. 2018. Drinking water microbiome assembly induced by water stagnation. ISME J. 12:1520. https://doi.org/10.1038/s41396-018-0101-5.

Malmuthuge, N., M. Li, Y. Chen, P. Fries, P. J. Griebel, B. Baurhoo, X. Zhao, and L. L. Guan. 2012. Distinct commensal bacteria associated with ingesta and mucosal epithelium in the gastrointestinal tracts of calves and chickens. FEMS Microbiol. Ecol. 79:337-347. https://doi.org/10.1111/j.1574-6941.2011.01220.x.

Malmuthuge, N., Y. Chen, G. Liang, L. A. Goonewardene, and L. L. Guan. 2015. Heat-treated colostrum feeding promotes beneficial bacteria colonization in the small intestine of neonatal calves. J. Dairy Sci. 98:8044-8053. https://doi.org/10.3168/jds.2015-9607.

Malmuthuge, N., J. P. Griebel, and L. L. Guan. 2014. Taxonomic identification of commensal bacteria associated with the mucosa and digesta throughout the gastrointestinal tract of pre-weaned calves. Appl. Environ. Microbiol. 80:2021-2028. https://doi.org/ 10.1128/AEM.03864-13.

Mao, S., R. Zhang, D. Wang, and W. Zhu. 2012. The diversity of the fecal bacterial community and its relationship with the concentration of volatile fatty acids in the feces during subacute rumen acidosis in dairy cows. BMC Vet. Res. 8:237. https://doi.org/10 .1186/1746-6148-8-237.

Marcobal, A., M. Barboza, E. D. Sonnenburg, N. Pudlo, E. C. Martens, P. Desai, C. B. Lebrilla, B. C. Weimer, D. A. Mills, J. B. German, and J. L. Sonnenburg. 2011. Bacteroides in the infant gut consume milk oligosaccharides via mucus-utilization pathways. Cell Host Microbe 10:507-514. https://doi.org/10.1016/j.chom 2011.10.007.

McGarvey, J. A., S. W. Hamilton, E. J. DePeters, and F. M. Mitloehner. 2010. Effect of dietary monensin on the bacterial population structure of dairy cattle colonic contents. Appl. Microbiol. Biotechnol. 85:1947-1952. https://doi.org/10.1007/s00253-009 -2229-8.

McMurdie, P. J., and S. Holmes. 2013. phyloseq: An R package for reproducible interactive analysis and graphics of microbiome census data. PLoS One 8:e61217. https://doi.org/10.1371/journal.pone .0061217 .

Meale, S. J., F. Chaucheyras-Durand, H. Berends, L. L. Guan, and M. A. Steele. 2017a. From pre- to postweaning: Transformation of the young calf's gastrointestinal tract. J. Dairy Sci. 100:5984-5995. https://doi.org/10.3168/jds.2016-12474.

Meale, S. J., S. Li, P. Azevedo, H. Derakhshani, J. C. Plaizier, E. Khafipour, and M. A. Steele. 2016. Development of ruminal and fecal microbiomes are affected by weaning but not weaning strategy in dairy calves. Front. Microbiol. 7:582. https://doi.org/10 .3389 /fmicb.2016.00582.

Meale, S. J., S. C. Li, P. Azevedo, H. Derakhshani, T. J. De Vries, J. C. Plaizier, M. A. Steele, and E. Khafipour. 2017b. Weaning age influences the severity of gastrointestinal microbiome shifts in dairy calves. Sci. Rep. 7:198. https://doi.org/10.1038/s41598-017 $-00223-7$.
Oikonomou, G., A. G. Teixeira, C. Foditsch, M. L. Bicalho, V. S. Machado, R. C. Bicalho, and Associations of Faecalibacterium Species with Health and Growth. 2013. Fecal microbial diversity in pre-weaned dairy calves as described by pyrosequencing of metagenomic 16S rDNA. Associations of Faecalibacterium species with health and growth. PLoS One 8:e63157. https://doi.org/10.1371/ journal.pone.0063157.

Owusu-Asiedu, A., J. F. Patience, B. Laarveld, A. G. Van Kessel, P. H. Simmins, and R. T. Zijlstra. 2006. Effects of guar gum and cellulose on digesta passage rate, ileal microbial populations, energy and protein digestibility, and performance of grower pigs. J. Anim. Sci. 84:843-852. https://doi.org/10.2527/2006.844843x.

Paggi, R. A., and J. P. Fay. 2004. Effect of short-chain acids on the carboxymethylcellulase activity of the ruminal bacterium Ruminococcus albus. Folia Microbiol. (Praha) 49:479-483. https://doi.org/ 10.1007/BF02931612.

Parada, A. E., D. M. Needham, and J. A. Fuhrman. 2016. Every base matters: Assessing small subunit rRNA primers for marine microbiomes with mock communities, time series and global field samples. Environ. Microbiol. 18:1403-1414. https://doi.org/10 $.1111 / 1462-2920.13023$.

Paz, H. A., K. E. Hales, J. E. Wells, L. A. Kuehn, H. C. Freetly, E. D. Berry, M. D. Flythe, M. L. Spangler, and S. C. Fernando. 2018. Rumen bacterial community structure impacts feed efficiency in beef cattle. J. Anim. Sci. 96:1045-1058. https://doi.org/10.1093/ jas/skx081.

Pereira, R. V., J. D. Siler, R. C. Bicalho, and L. D. Warnick. 2014 Multiresidue screening of milk withheld for sale at dairy farms in central New York State. J. Dairy Sci. 97:1513-1519. https://doi .org/10.3168/jds.2013-7421.

Pereira, R. V. V., S. Lima, J. D. Siler, C. Foditsch, L. D. Warnick, and R. C. Bicalho. 2016. Ingestion of milk containing very low concentration of antimicrobials: Longitudinal effect on fecal microbiota composition in preweaned calves. PLoS One 11:e0147525. https:// doi.org/10.1371/journal.pone.0147525.

Pruesse, E., C. Quast, K. Knittel, B. M. Fuchs, W. Ludwig, J. Peplies, and F. O. Glöckner. 2007. SILVA: A comprehensive online resource for quality checked and aligned ribosomal RNA sequence data compatible with ARB. Nucleic Acids Res. 35:7188-7196. https:// doi.org/10.1093/nar/gkm864.

Reese, A. T., and R. R. Dunn. 2018. Drivers of microbiome biodiversity: A review of general rules, feces, and ignorance. MBio 9:e01294-e18. https://doi.org/10.1128/mBio.01294-18.

Sasada, T., T. Hinoi, Y. Saito, T. Adachi, Y. Takakura, Y. Kawaguchi, Y. Sotomaru, K. Sentani, N. Oue, W. Yasui, and H. Ohdan. 2015. Chlorinated water modulates the development of colorectal tumors with chromosomal instability and gut microbiota in Apcdeficient mice. PLoS One 10:e0132435. https://doi.org/10.1371/ journal.pone.0132435.

Shanks, O. C., C. A. Kelty, S. Archibeque, M. Jenkins, R. J. Newton, S. L. McLellan, S. M. Huse, and M. L. Sogin. 2011. Community structures of fecal bacteria in cattle from different animal feeding operations. Appl. Environ. Microbiol. 77:2992-3001. https://doi .org/10.1128/AEM.02988-10.

Shannon, M. C., and G. M. Hill. 2019. Trace mineral supplementation for the intestinal health of young monogastric animals. Front. Vet. Sci. 6:73. https://doi.org/10.3389/fvets.2019.00073.

Simmering, R., and M. Blaut. 2001. Pro- and prebiotics - The tasty guardian angels? Appl. Microbiol. Biotechnol. 55:19-28. https:// doi.org/10.1007/s002530000512.

Soberon, F., E. Raffrenato, R. W. Everett, and M. E. Van Amburgh. 2012. Preweaning milk replacer intake and effects on long-term productivity of dairy calves. J. Dairy Sci. 95:783-793. https://doi .org/10.3168/jds.2011-4391.

Sofi, M. H., R. Gudi, S. Karumuthil-Melethil, N. Perez, B. M. Johnson, and C. Vasu. 2014. pH of drinking water influences the composition of gut microbiome and type 1 diabetes incidence. Diabetes 63:632-644. https://doi.org/10.2337/db13-0981.

Strunz, U. T., and M. I. Grossman. 1978. Effect of intragastric pressure on gastric emptying and secretion. Am. J. Physiol. 235:E552E555. 
Tempini, P. N., S. S. Aly, B. M. Karle, and R. V. Pereira. 2018. Multidrug residues and antimicrobial resistance patterns in waste milk from dairy farms in Central California. J. Dairy Sci. 101:81108122. https://doi.org/10.3168/jds.2018-14398.

USDA. 2016. Dairy 2014, Dairy Cattle Management Practices in the United States, 2014. USDA-APHIS:VS-CEAH-NAHMS, Fort Collins, CO.

Van De Stroet, D. L., J. A. Calderón Díaz, K. J. Stalder, A. J. Heinrichs, and C. D. Dechow. 2016. Association of calf growth traits with production characteristics in dairy cattle. J. Dairy Sci. 99:8347-8355. https://doi.org/10.3168/jds.2015-10738.

Vaughan, E. E., M. C. de Vries, E. G. Zoetendal, K. Ben-Amor, A. D. Akkermans, and W. M. de Vos. 2002. The intestinal LABs. Antonie van Leeuwenhoek 82:341-352. https://doi.org/10.1023/A: 1020672724450.

Vlková, E., V. Rada, D. Bujnáková, and V. Kmet. 2004. Enumeration, isolation, and identification of Bifidobacteria from infant feces. Folia Microbiol. (Praha) 49:209-212. https://doi.org/10.1007/ BF02931404.

Wickramasinghe, H. K. J. P., A. J. Kramer, and J. A. D. R. N. Appuhamy. 2019. Drinking water intake of newborn dairy calves and its effects on feed intake, growth performance, health status, and nutrient digestibility. J. Dairy Sci. 102:377-387. https://doi.org/ $10.3168 /$ jds.2018-15579.
Xi, C., Y. Zhang, C. F. Marrs, W. Ye, C. Simon, B. Foxman, and J. Nriagu. 2009. Prevalence of antibiotic resistance in drinking water treatment and distribution systems. Appl. Environ. Microbiol. 75:5714-5718. https://doi.org/10.1128/AEM.00382-09.

Yáñez-Ruiz, D. R., L. Abecia, and C. J. Newbold. 2015. Manipulating rumen microbiome and fermentation through interventions during early life: A review. Front. Microbiol. 6:1133. https://doi.org/10 $.3389 /$ fmicb. 2015.01133.

Yeoman, C. J., S. L. Ishaq, E. Bichi, S. K. Olivo, J. Lowe, and B. M. Aldridge. 2018. Biogeographical differences in the influence of maternal microbial sources on the early successional development of the bovine neonatal gastrointestinal tract. Sci. Rep. 8:3197. https: //doi.org/10.1038/s41598-018-21440-8.

\section{ORCIDS}

H. K. J. P. Wickramasinghe ๑ https://orcid.org/0000-0002-9493-1513

J. M. Anast () https://orcid.org/0000-0003-4701-9342

S. Schmitz-Esser @ https://orcid.org/0000-0002-1907-0709

J. A. D. R. N. Appuhamy @ https://orcid.org/0000-0001-8767-0303 